\title{
Correlation between vascular endothelial growth factor and long-term prognosis in patients with acute myocardial infarction
}

\author{
JIAMIN NIU, XIA HAN, HUAXIN QI, JIE YIN, ZHIQIANG ZHANG and ZENGTANG ZHANG \\ Department of Cardiology, Laiwu People's Hospital, Laiwu, Shandong 271100, P.R. China
}

Received October 27, 2014; Accepted November 5, 2015

DOI: $10.3892 /$ etm.2016.3286

\begin{abstract}
The aim of the present study was to investigate the correlation between plasma the levels of vascular endothelial growth factor (VEGF) and major adverse cardiovascular events (MACE) in patients with acute myocardial infarction (AMI). A total of 124 patients with AMI undergoing emergency percutaneous coronary intervention (PCI) were selected, and plasma VEGF levels were measured 7 days after the onset of AMI using an enzyme-linked immunosorbent assay. The patients were divided into the $\mathrm{L}(\leq 190 \mathrm{pg} / \mathrm{ml}$ VEGF) and H (>190 pg/ml VEGF) groups, and were followed up every 2 months for an average of 12 months. MACE were recorded during follow-up. On the basis of these results, the patients were further divided into the MACE and non-MACE (N-MACE) groups, and the serum VEGF concentration was compared between the two groups. At the 6-month follow-up, the incidence of MACE in the $\mathrm{H}$ group was found to be significantly reduced compared with the $\mathrm{L}$ group. The serum VEGF concentration in the N-MACE group was significantly higher compared with the MACE group. Multinomial logistic regression revealed that reduced VEGF levels $(\beta=1.243$; 95\% CI, 1.018-1.326; $\mathrm{P}=0.026$ ) were independent risk factors for MACE. In conclusion, high plasma VEGF levels at 7 days after AMI onset facilitate the long-term prognosis in the same infarct zone in patients with AMI, while low plasma VEGF levels are independent risk factors for MACE.
\end{abstract}

\section{Introduction}

Vascular endothelial growth factor (VEGF) is a signal protein produced by endothelial cells, smooth muscle cells and macrophages, which is able to increase vascular permeability, stimulate the expression of vascular endothelial cells and induce angiogenesis (1). Independently of the physiological

Correspondence to: Dr Zengtang Zhang, Department of Cardiology, Laiwu People's Hospital, 1 Xuehu Street, Laiwu, Shandong 271100, P.R. China

E-mail: zengtangzhang@126.com

Key words: acute myocardial infarction, vascular endothelial growth factor, long-term prognosis or pathological state, VEGF is crucially involved in vascular endothelial function repairing and vascular regeneration $(2,3)$. Previous studies have demonstrated that plasma VEGF levels are significantly elevated in patients with acute myocardial infarction (AMI) compared with those in healthy individuals (4-7). Furthermore, studies involving AMI in animal models $(8,9)$ have shown that VEGF promotes angiogenesis in infarct regions and reduces AMI area. Yin et al (10) and Hojo et al (11) observed that hypoxia and ischemia stimulate VEGF secretion in patients with AMI, and suggested that the marked increase in VEGF indicates a protective effect on patients as a result of angiogenesis and endothelial cell proliferation. According to its biological effects, VEGF may be able to improve the long-term prognosis of patients with AMI (11). However, the correlation between plasma VEGF levels and long-term prognosis in patients with AMI remains controversial. Matsudaira et al (12) observed that low plasma VEGF levels following the onset of AMI are associated with a significantly increased risk for MACE during 6 months of follow-up. In addition, Heeschen et al (13) indicated that high VEGF levels following the onset of acute coronary syndrome (ACS) are associated with a poor outcome. Therefore, in the present study, the correlation between plasma VEGF levels in the same infarct zone at 7 days after the onset of AMI following successful revascularization by percutaneous coronary intervention $(\mathrm{PCI})$ and the long-term prognosis were evaluated in patients with AMI.

\section{Materials and methods}

Subjects. A total of 124 patients (76 females and 48 males; mean age, 59.1 years) with AMI were recruited from Laiwu People's Hospital (Laiwu, China) between June 2010 and February 2014. The present study was conducted in accordance with the declaration of Helsinki and was approved by the Ethics Committee of the Laiwu People's Hospital. Written informed consent was obtained from all participants.

Inclusion criteria. According to the standard diagnosis for AMI $(14,15)$, patients were required to meet minimum two out of the three of the following criteria to be included in the study: i) Clinical history of ischemic chest pain; ii) dynamic electrocardiogram (ECG) changes; iii) dynamic changes in plasma levels of markers of myocardial necrosis. Furthermore, according to coronary artery segmentation (16), lesions involve 
segment 6 of left anterior descending (LAD) artery but not in left circumflex and right coronary arteries. In addition, PCI and stent implantation performed within $12 \mathrm{~h}$ from symptom onset to achieve a thrombolysis in MI (TIMI) flow grade 3.

Exclusion criteria. Patients were excluded if they had a history of any of the following: i) Angina following PCI; ii) previous MI indicated by past medical history, ECG or Color Doppler echocardiography; iii) prior PCI or coronary artery bypass grafting; iv) history of heart failure; v) left ventricular hypertrophy; vi) atrial fibrillation; vii) pacemaker implantation; viii) renal insufficiency; ix) digitalis administration; $\mathrm{x})$ valvular heart disease; $\mathrm{xi}$ ) severe lung disease; $\mathrm{xii}$ ) acute or chronic infection; xiii) anemia; xiv) acute or chronic liver diseases, such as chronic hepatitis and liver cirrhosis; $\mathrm{xv}$ ) cancer; $\mathrm{xvi}$ ) organ or bone marrow transplantation.

Control subjects. A total of 30 subjects were recruited into the control group, over the same time period from the same hospital. Subjects in the control group were selected according to the following criteria: i) No hypertension or diabetes or other organic diseases; ii) normal blood routine, liver and kidney function test results, and normal abdomen and cardiac ultrasound, ECG and chest radiograph; and iii) no cancer or pregnancy.

Treatment. According to the guidelines for the management of patients with ST-elevation MI $(14,15)$, all the patients received conventional drug therapy, and underwent PCI and stent implantation (EXCEL drug-eluting stent; JW Medical Systems Co., Weihai, China) within $12 \mathrm{~h}$ following symptom onset, to achieve thrombolysis in TIMI flow grade 3 . The conventional drug therapy involved administration of aspirin enteric-coated tablets (100 mg orally, once a day; Bayer Health Care AG, Beijing, China), clopidogrel hydrogen sulphate tablets $(75 \mathrm{mg}$ orally, once a day; Sanofi Pharmaceuticals Co., Ltd., Hangzhou, China), rosuvastatin calcium tablets (10 mg orally, once a day; AstraZeneca Pharmaceutical Co., Ltd., Wuxi, China), metoprolol succinate sustained-release tablets $(11.875 \mathrm{mg}$ orally, once a day; AstraZeneca Pharmaceutical Co., Ltd.).

VEGF measurement. Fasting venous blood samples (2 ml) were obtained from patients 7 days after the onset of AMI and centrifuged at 3,000 rpm for $10 \mathrm{~min}$. Plasma was isolated and stored at $-20^{\circ} \mathrm{C}$ for further analysis. VEGF levels were determined using VEGF-specific enzyme-linked immunosorbent assay (R\&D Systems, Minneapolis, MN, USA), and measurements were performed according to the manufacturer's instructions. The antiserum containing the mouse anti-human monoclonal VEGF antibody (cat. no. MAB286; dilution, 1:100; R\&D Systems) was adsorbed into the wells of a microtiter plate (BrandTech Scientific, Inc., Essex, CT, USA) and then washed three times with phosphate-buffered saline. Next, the blood sample was added, and binding occurred if the antiserum antibody matched the blood sample antigen. Subsequently, the excessive antibody was washed away, and the rabbit anti-mouse IgG secondary antibody (cat. no. G-202-C; dilution 1:400; R\&D Systems) was added, which would specifically react with the test antigen to form the 'sandwich'. Tetramethyl benzidine (Sigma-Aldrich, St. Louis, MO,
USA) was then added and the production of colored enzymatic products indicated that the corresponding antigen was present on the well walls. The optical density of the end-product was measured using an F-7000 fluorescence spectrophotometer (Hitachi High-Technologies Corp., Tokyo, Japan).

Echocardiography. Echocardiography was performed to determine left ventricular ejection fraction (LVEF) and evaluate the cardiac systolic function in patients with AMI during hospital stay and follow-ups, as well as 1, 6 and 12 months after PCI. A Vivid E9 Doppler ultrasonic scanner (GE Healthcare Life Sciences, Shanghai, China) was used at a scanning speed of $50 \mathrm{~mm} / \mathrm{sec}$ and frequency of 2.0-2.5 MHz. The left ventricular end systolic and diastolic volumes (LVESV and LVEDV, respectively) were measured according to the modified Simpson's rule using the apical two-chamber and four-chamber views recommended by the American Society of Echocardiography (17). LVEF = (LVEDV-LVESV)/LVEDV. An LVEF of $<40 \%$ was diagnosed as heart failure.

Follow-up. All patients with AMI were followed up every 2 months for an average of 12 months. The endpoint of the study was the incidence of major adverse cardiovascular events (MACE) during hospitalization period or follow-up, including cardiac mortality, heart failure, severe arrhythmia, cardiac shock and post-infarction angina.

Statistical analysis. Statistical analysis was performed using SPSS software, version 19.0 (IBM SPSS, Armonk, NY, USA). Data were presented as mean \pm standard deviation and were analyzed using the t-test and $\chi^{2}$ test. VEGF was numerical variable following skewed distribution and presented as a median. The Wilcoxon rank-sum test was employed for the comparison of two independent samples. The comparison among multiple samples was performed using the $\chi^{2}$ test. $\mathrm{P}<0.05$ was considered to indicate a statistically significant difference.

\section{Results}

General patient data and VEGF levels. According to their plasma VEGF levels, the patients were divided into the $\mathrm{L}(\leq 190 \mathrm{pg} / \mathrm{ml})$ and $\mathrm{H}(>190 \mathrm{pg} / \mathrm{ml})$ groups. General data and plasma VEGF levels of all groups are presented in Table I. No significant differences were identified in the general data of patients, including gender, age, systolic blood pressure (SBP), body mass index (BMI), presence of diabetes, smoking habits, and angiotensin-converting enzyme inhibitors (ACEI) and $\beta$-blocker administration among the control, $\mathrm{H}$ and $\mathrm{L}$ groups ( $P>0.05)$. By contrast, the concentrations of serum VEGF in the $\mathrm{H}$ and $\mathrm{L}$ groups was significantly different compared with control group $(\mathrm{P}<0.05)$.

Follow-up. At the 12-month follow-up, 18/124 patients with MI presented with MACE, including 2 cases of mortality, 7 cases of heart failure and 9 cases of recurrent angina or MI.

Correlation between VEGF levels and clinical outcomes. Based on the incidence of MACE, the 124 patients were divided into the MACE $(n=18)$ and non-MACE (N-MACE; $n=106)$ groups. The median plasma VEGF levels in the MACE group were 
Table I. General data and plasma VEGF levels among the three groups.

\begin{tabular}{|c|c|c|c|c|}
\hline Characteristic & Control group $(n=30)$ & $H$ group $(n=62)$ & L group $(n=62)$ & P-value \\
\hline Males, n & 18 & 25 & 23 & 0.100 \\
\hline Age, years & $57.3 \pm 8.7$ & $59.3 \pm 9.8$ & $58.9 \pm 8.8$ & 0.683 \\
\hline $\mathrm{SBP}, \mathrm{mmHg}$ & $145 \pm 21$ & $156 \pm 18$ & $150 \pm 20$ & 0.248 \\
\hline Diabetes, $\mathrm{n}$ & 4 & 13 & 19 & 0.156 \\
\hline Smoking, $\mathrm{n}$ & 12 & 20 & 18 & 0.574 \\
\hline BMI & $25.5 \pm 2.1$ & $27.8 \pm 3.0$ & $26.1 \pm 2.3$ & 0.053 \\
\hline ACEI administration, $\mathrm{n}$ & 10 & 18 & 16 & 0.751 \\
\hline$\beta$-blocker administration, $\mathrm{n}$ & 5 & 10 & 8 & 0.843 \\
\hline Median VEGF, pg/ml & 82.15 & 265.42 & 121.60 & 0.033 \\
\hline
\end{tabular}

SBP, systolic blood pressure; VEGF, vascular endothelial growth factor; BMI, body mass index; ACEI, angiotensin-converting enzyme inhibitors; L group, $\leq 190$ pg/ml VEGF plasma level; H group, >190 pg/ml VEGF plasma level; VEGF, vascular endothelial growth factor.

Table II. Incidence of major adverse cardiovascular events in the $\mathrm{H}$ and $\mathrm{L}$ groups ( $\mathrm{n}=62$ per group).

\begin{tabular}{lccc}
$\begin{array}{l}\text { Time after } \\
\text { AMI (months) }\end{array}$ & H group, \% (n) & L group, \% (n) & P-value \\
\hline 0 & $0.0(0)$ & $0.0(0)$ & - \\
2 & $0.0(0)$ & $3.2(2)$ & 0.154 \\
4 & $3.2(2)$ & $8.1(5)$ & 0.243 \\
6 & $3.2(2)$ & $12.9(8)$ & 0.048 \\
8 & $4.8(3)$ & $16.1(10)$ & 0.040 \\
10 & $6.5(4)$ & $19.4(12)$ & 0.032 \\
12 & $8.1(5)$ & $21.0(13)$ & 0.041
\end{tabular}

AMI, acute myocardial infarction; L group, $\leq 190 \mathrm{pg} / \mathrm{ml}$ VEGF plasma level; H group, >190 pg/ml VEGF plasma level; VEGF, vascular endothelial growth factor.

found to be significantly reduced $(153.23 \mathrm{pg} / \mathrm{ml})$ compared with those in the N-MACE group (208.05 pg/ml; $\mathrm{P}<0.001)$.

Incidence of MACE. At 6 months after the onset of AMI, the incidence of MACE was markedly higher in the L group compared with the $\mathrm{H}$ group; MACE-free survival rate was markedly higher in the $\mathrm{H}$ group compared with the $\mathrm{L}$ group (Table II and Fig. 1).

Logistic regression analysis. Logistic regression analysis was performed with MACE as the dependent variable (yes=1, no=0) and gender, age, SBP, diabetes, smoking, BMI, ACEI and $\beta$-blocker administration and VEGF levels as the independent variables. The results suggested that a low VEGF level $(\leq 190 \mathrm{pg} / \mathrm{ml})$ was an independent risk factor for MACE $(\beta=1.243 ; 95 \% \mathrm{CI}, 1.018-1.326 ; \mathrm{P}=0.026)$.

\section{Discussion}

The present study involved 124 patients with AMI with single-vessel, proximal LAD disease, in which

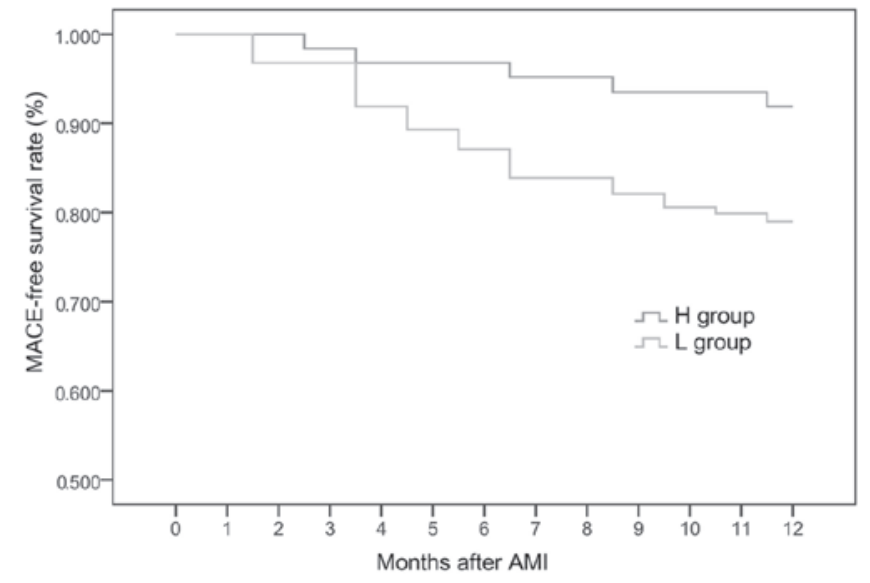

Figure 1. MACE-free survival rates among the two groups. The MACE-free survival rate of patients with acute myocardial infarction was significantly higher in the H group compared with that in the L group, when the follow-up was longer than 6 months. MACE, major adverse cardiovascular events; AMI, acute myocardial infarction; L group, $\leq 190 \mathrm{pg} / \mathrm{ml}$ VEGF plasma level; H group, $>190$ pg/ml VEGF plasma level; VEGF, vascular endothelial growth factor.

revascularization was performed within $12 \mathrm{~h}$. Plasma VEGF levels were evaluated at 7 days after the onset of AMI. At the 12-month follow-up, the incidence of MACE in the patients, including cardiac mortality, heart failure, severe arrhythmia, cardiac shock and post-infarction angina was recorded. The results showed that the incidence of MACE in the $\mathrm{H}$ group was significantly higher compared with the L group at the 6-12-month follow-ups, and that the plasma VEGF levels were markedly elevated in the N-MACE group, as compared with those in the MACE group. The correlation between plasma VEGF levels and long-term prognosis in patients with AMI was investigated using logistic regression analysis, which revealed that a low VEGF level $(\beta, 1.243 ; 95 \%$ CI, 1.018-1.326; $\mathrm{P}=0.026$ ) was an independent risk factor for MACE, while high VEGF levels facilitated long-term prognosis in patients with AMI.

VEGF is a dimeric glycoprotein composed of two identical polypeptide chains, which is produced by endothelial cells, smooth muscle cells and macrophages. VEGF is able to promote 
vascular endothelial cell mitosis, proliferation and migration, increase vascular permeability, stimulate the expression of vascular endothelial cells and induce angiogenesis (1). VEGF expression has been shown to be promoted by several factors, including hypoxia, angiotensin, advanced glycation end products, endotoxin and high glucose $(18,19)$. VEGF is known to be crucially involved in the induction of vasculogenesis and angiogenesis, exerting a marked effect on angiogenesis by binding to kinase insert domain-containing receptor (20). Furthermore, VEGF may exert a positive effect in the revascularization of ischemic tissues, specifically through the promotion of vascular endothelial cell proliferation and neovascularization, increase of vascular permeability, and improvement of collateral circulation (20). Sluimer et al (21) performed carotid endarterectomy in patients with carotid atherosclerosis and observed an increase in the mRNA and protein expression of hypoxia-inducible factor 1 (HIF-1 $\alpha$ ), VEGF expression and microvessel density in the early stages of atherosclerosis. In addition, hypoxia correlated with thrombosis, angiogenesis, HIF- $1 \alpha$ and VEGF expression; therefore, VEGF and HIF-1 $\alpha$ were associated with atherosclerotic plaque instability and were considered to be an index indicating plaque instability in patients with ACS. Mao et al (22) used a rat model of myocardial ischemia and ligated the left descending coronary artery. After $3 \mathrm{~h}$ the VEGF expression in the ischemic myocardium had peaked, subsequently decreasing $6 \mathrm{~h}$ later. Thus, the VEGF expression level was able to assist in determining the degree of severity of ischemic injury. Wang et al (23) showed that short-term intermittent hypoxia has a cardioprotective effect, which may result from the upregulation of VEGF and HIF-1 $\alpha$ expression in a rat model of ischemia/reperfusion injury. In 2003, Heeschen et al (13) measured the plasma VEGF levels of 1,090 patients with ACS at $8.7 \mathrm{~h}$ after the onset of MI, and observed that the incidence of MACE was increased in patients that exhibited significantly elevated plasma VEGF levels at the 6-month follow-up. In 2012, Matsudaira et al (12) detected the plasma VEGF levels in 873 patients with AMI 7 days after the onset of MI and found that the incidence of MACE was higher in patients with low plasma VEGF levels $(\leq 190 \mathrm{pg} / \mathrm{ml})$ at the 6-month follow-up. The present study included patients with AMI with single-vessel, proximal LAD disease, with a similar myocardial ischemia and injury area, in order to more accurately indicate the association between VEGF and MACE. In 2012, Devaux et al (24) recruited 290 patients with AMI and revealed that the risk of left ventricular remodeling was higher in patients with lower VEGF levels. Zentilin et al (25) reported that the long-term expression of VEGF-A165 and VEGF-B167 in the myocardium may reduce myocardial fibrosis and enhance myocardial contractility to protect the viable myocardium and improve ventricular remodeling. Furthermore, previous studies have indicated that the plasma VEGF levels in patients with AMI peaked on days 7-14 after the onset of AMI, and in patients that underwent successful percutaneous revascularization on day 7 (26-28). Collectively, the aforementioned and present results indicate that the increase in VEGF levels serves a crucial function in cardiovascular repair.

In addition, the results of the aforementioned studies suggest that due to the ischemia and hypoxia stimulation, patients with AMI may be expected to secrete increased quantities of VEGF to promote the angiogenesis and endothelial cell proliferation, therefore promoting self-repair and cardiac remodeling, which indicates the cardioprotective effect of VEGF expression. In the present study, increased VEGF levels were shown to facilitate long-term prognosis in patients with AMI, while low VEGF levels were found to be independent risk factors for MACE, which is consistent with the results from the study by Matsudaira et al (12). By contrast, Shimokawahara et al (29) observed that in the acute period, the left ventricle volume index of the high-serum-VEGF-concentration group of patients with MI was significantly elevated compared with the low-serum-VEGF-concentration group, while the intergroup differences observed in the chronic period were not statistically significant. In conclusion, high plasma VEGF levels at 7 days after AMI onset facilitate the long-term prognosis in the same infarct zone in patients with AMI, while low plasma VEGF levels are independent risk factors for MACE. However, the correlation between plasma VEGF levels at different stages, genetic factors and prognosis in patients with AMI requires further investigation.

\section{Acknowledgements}

This study was supported by a grant from the Shandong Science and Technology Development in Health and Medical Care (grant no. 2013WS0072).

\section{References}

1. Giatromanolaki A, Sivridis E, Athanassou N, Zois E, Thorpe PE, Brekken RA, Gatter KC, Harris AL, Koukourakis IM and Koukourakis MI: The angiogenic pathway 'vascular endothelial growth factor/flk-1(KDR)-receptor' in rheumatoid arthritis and osteoarthritis. J Pathol 194: 101-108, 2001.

2. Gerber HP, McMurtrey A, Kowalski J, Yan M, Keyt BA, Dixit V and Ferrara N: Vascular endothelial growth factor regulates endothelial cell survival through the phosphatidylinositol 3'-kinase/Akt signal transduction pathway: Requirement for Flk-1/KDR activation. J Biol Chem 273: 30336-30343, 1998.

3. Deuse T, Peter C, Fedak PW, Doyle T, Reichenspurner H, Zimmermann WH, Eschenhagen T, Stein W, Wu JC, Robbins RC and Schrepfer S: Hepatocyte growth factor or vascular endothelial growth factor gene transfer maximizes mesenchymal stem cell-based myocardial salvage after acute myocardial infarction. Circulation 120 (Suppl 11): S247-S254, 2009.

4. Soeki T, Tamura Y, Shinohara H, Tanaka H, Bando K and Fukuda N: Serial changes in serum VEGF and HGF in patients with acute myocardial infarction. Cardiology 93: 168-174, 2000.

5. Ogawa H, Suefuji H, Soejima H, Nishiyama K, Misumi K, Takazoe K, Miyamoto S, Kajiwara I, Sumida H, Sakamoto T, et al: Increased blood vascular endothelial growth factor levels in patients with acute myocardial infarction. Cardiology 93: 93-99, 2000.

6. Lee KW, Lip GY and Blann AD: Plasma angiopoietin-1, angiopoietin-2, angiopoietin receptor tie-2 and vascular endothelial growth factor levels in acute coronary syndromes. Circulation 110: 2355-2360, 2004.

7. Soeki T, Tamura Y, Shinohara H, Sakabe K, Onose Y and Fukuda N: Serum hepatocyte growth factor predicts ventricular remodeling following myocardial infarction. Circ J 66: 1003-1007, 2002.

8. Hagikura K, Fukuda N, Yokoyama S, Yuxin L, Kusumi Y, Matsumoto T, Ikeda Y,Kunimoto S, Takayama T, Jumabay M, et al: Low invasive angiogenic therapy for myocardial infarction by retrograde transplantation of mononuclear cells expressing the VEGF gene. Int J Cardiol 142: 56-64, 2010.

9. Ye L, Zhang W, Su LP, Haider HK, Poh KK, Galupo MJ, Songco G, Ge RW, Tan HC and Sim EK: Nanoparticle based delivery of hypoxia-regulated VEGF transgene system combined with myoblast engraftment for myocardial repair. Biomaterials 32: 2424-2431, 2011 
10. Yin R, Feng J, Chen D and Wu H: Serum levels of vascular endothelial growth factor in patients with anginapectoris and acute myocardial infarct. Chin Med Sci J 15: 205-209, 2000.

11. Hojo Y, Ikeda U, Zhu Y, Okada M, Ueno S, Arakawa H, Fujikawa H, Katsuki T and Shimada K: Expression of vascular endothelial growth factor in patients with acute myocardial infarct. J Am Coll Cardiol 35: 968-973, 2000.

12. Matsudaira K, Maeda K, Okumura N, Yoshikawa D, Morita Y, Mitsuhashi H, Ishii H, Kondo T and Murohara T; Nagoya Acute Myocardial Infarction Study (NAMIS) Group: Impact of low levels of vascular endothelial growth factor after myocardial infarction on 6-month clinical outcome: Results from the Nagoya Acute Myocardial Infarction Study. Circ J 76: 1509-1516, 2012.

13. Heeschen C, Dimmeler S, Hamm CW, Boersma E, Zeiher AM and Simoons ML; CAPTURE (c7E3 Anti-Platelet Therapy in Unstable REfractory angina) Investigators: Prognostic significance of angiogenic growth factor serum levels in patients with acute coronary syndromes. Circulation 107: 524-530, 2003.

14. Canadian Cardiovascular Society; American Academy of Family Physicians; American College of Cardiology; American Heart Association; Antman EM, Hand M, Armstrong PW, Bates ER, Green LA, Halasyamani LK, Hochman JS, Krumholz HM, Lamas GA, Mullany CJ, et al: 2007 focused update of the ACC/AHA 2004 guidelines for the management of patients with ST-elevation myocardial infarction: A report of the American College of Cardiology/American Heart Association Task Force on Practice Guidelines. J Am Coll Cardiol 51: 210-247, 2008.

15. Braunwald E, Antman EM, Beasley JW, Califf RM, Cheitlin MD, Hochman JS, Jones RH, Kereiakes D, Kupersmith J, Levin TN, et al; American College of Cardiology; American Heart Association. Committee on the Management of Patients With Unstable Angina: ACC/AHA 2002 guideline update for the management of patients with unstable angina and non-ST-segment elevation myocardial infarction - summary article: A report of the American College of Cardiology/American Heart Association task force on practice guidelines (Committee on the Management of Patients with Unstable Angina). J Am Coll Cardiol 40: 1366-1374, 2002

16. Austen WG, Edwards JE, Frye RL, Gensini GG, Gott VL, Griffith LS, McGoon DC, Murphy ML and Roe BB: A reporting system on patients evaluated for coronary artery disease. Report of the Ad Hoc Committee for Grading of Coronary Artery Disease, Council on Cardiovascular Surgery, American Heart Association. Circulation 51 (Suppl 4): 5-40, 1975.

17. Poulsen SH: Clinical aspects of left ventricular diastolic function assessed by Doppler echocardiography following acute myocardial infarction. Dan Med Bull 48: 199-210, 2001.
18. Bates DO, Hillman NJ, Williams B, Neal CR and Pocock TM: Regulation of microvascular permeability by vascular endothelial growth factors. J Anat 200: 581-597, 2002.

19. Maeda K, Chung YS, Ogawa Y, Takatsuka S, Kang SM, Ogawa M, Sawada T and Sowa M: Prognostic value of vascular endothelial growth factor expression in gastric carcinoma. Cancer 77: 858-863, 1996.

20. Hoberg M, Schmidt EL, Tuerk M, Stark V, Aicher WK and Rudert M: Induction of endostatin expression in meniscal fibrochondrocytes by co-culture with endothelial cells. Arch Orthop Trauma Surg 129: 1137-1143, 2009.

21. Sluimer JC, Gasc JS, van Wanroij JL, Kisters N, Groeneweg M, Sollewijn Gelpke MD, Cleutjens JP, van den Akker LH, Corvol P, Wouters BG, et al: Hypexia, hypoxia-inducible transcription factor, and macrophages in human atherosclerotic plaques are correlated with intraplaque angiogenesis. J Am Coll Cardial 51: 1258-1265, 2008

22. Mao RM, Du ZB, Gao WM, Mi L and Zhu BL: Time-dependent expression of vascular endothelial growth factor after acute myocardial ischemia in rats. Fa Yi Xue Za Zhi 28: 179-184, 2012 (In Chinese).

23. Wang $\mathrm{Z}$ and Si LY: Hypoxia-inducible factor-1 $\alpha$ and vascular endothelial growth factor in the cardioprotective effects of intermittent hypoxia in rats. Ups J Med Sci 118: 65-74, 2013.

24. Devaux Y, Vausort M, Azuaje F, Vaillant M, Lair ML, Gayat E, Lassus J, Ng LL, Kelly D, Wagner DR and Squire IB: Low levels of vascular endothelial growth factor B predict left ventricular remodeling after acute myocardial infarction. J Card Fail 18: 330-337, 2012.

25. Zentilin L, Puligadda U, Lionetti V, Zacchigna S, Collesi C, Pattarini L, Ruozi G, Camporesi S, Sinagra G, Pepe M, et al: Cardiomyocyte VEGFR-1 activation by VEGF-B induces compensatory hypertrophy and preserves cardiac function after myocardial infarction. FASEB J 24: 1467-1478, 2010.

26. Ferrara N, Gerber HP and LeCouter J: The biology of VEGF and its receptors. Nat Med 9: 669-676, 2003.

27. Gruchala M, Roy H, Bhardwaj S and Ylä-Herttuala S: Gene therapy for cardiovascular diseases. Curr Pharm Des 10: 407-423, 2004

28. Ratner M: Genentech discloses safety concerns over Avastin. Nat Biotechnol 22: 1198, 2004.

29. Shimokawahara H, Jougasaki M, Setoguchi M, Ichiki T, Sonoda M, Nuruki N, Nakashima H, Murohara T and Tsubouchi H: Relationship between vascular endothelial growth factor and left ventricular dimension in patients with acute myocardial infarction. J Cardiol 64: 360-365, 2014. 